Rev. Int. Contam. Ambie. 35 (4) 979-989, 2019

DOI: 10.20937/RICA.2019.35.04.16

\title{
EFECTO DE LA FRECUENCIA DE VOLTEO EN EL BIOSECADO DE RESIDUOS SÓLIDOS ORGÁNICOS
}

Effect of the turning frequency in the biodrying of organic solid waste

\author{
Carlos OROZCO ÁLVAREZ ${ }^{1}$, Javier DÍAZ MEGCHÚN ${ }^{1}$, \\ Manuel de Jesús MACÍAS HERNÁNDEZ ${ }^{2}$ y Fabián ROBLES MARTÍNEZ ${ }^{1 *}$
}

${ }^{1}$ Instituto Politécnico Nacional, Unidad Profesional Interdisciplinaria de Biotecnología, Av. Acueducto s/n, Barrio La Laguna-Ticomán, Delegación Gustavo A. Madero, 07340 Ciudad de México, México

${ }^{2}$ Instituto Politécnico Nacional, Escuela Superior de Ingeniería Química e Industrias Extractivas, Unidad Profesional Adolfo López Mateos. Colonia Lindavista, Delegación Gustavo A. Madero, 07738 Ciudad de México, México

*Autor para correspondencia: froblesm@ipn.mx

(Recibido: enero 2018; aceptado: noviembre 2018)

Palabras clave: actividad microbiana, tiempo de proceso, biorresiduos

\section{RESUMEN}

En este trabajo se evaluó el efecto de la frecuencia de volteo en el biosecado de tres pilas, las cuales fueron mantenidas dentro de una estructura tipo invernadero. Las pilas estuvieron compuestas de una mezcla de cáscara de naranja (56\%), mantillo (25\%) y pasto $(19 \%)$. La frecuencia de volteo fue de cada 10 días para la pila 1 , cada seis días para la pila 2 y cada tres días para la pila 3 . En el centro de la pila 3 se alcanzó una humedad de $26.87 \%$ en 28 días, mientras que en las pilas 2 y 1 se obtuvo una humedad similar a la pila 3 pero hasta los 34 y 37 días, respectivamente. A los 40 días de proceso, el contenido de humedad en el centro de las tres pilas se estabilizó en aproximadamente $20 \%$, mientras que en la superficie de las mismas fue de $10 \%$. La deshidratación en la pila 3 fue más rápida y la actividad microbiana de fase termofílica duró ocho días menos que en los otros dos tratamientos. El calor de combustión del material de la pila 3 fue de $15852.67 \mathrm{~kJ} / \mathrm{kg}$, por lo tanto tiene la calidad de residuos para ser combustionados de acuerdo con las especificaciones de la European Union for Responsible Incineration and Treatment of Special Waste. En consecuencia, el tiempo más corto para biosecar los residuos orgánicos se presentó en la pila 3, que fue la pila que tuvo la mayor frecuencia de volteo.

Key words: microbian activity, time process, biowaste

\begin{abstract}
In this work the frequency of turning in the biodrying of three piles composed of a mixture of orange shells $(56 \%)$, mulch $(25 \%)$ and grass $(19 \%)$ inside a greenhouse was evaluated. The turning period was 10 , six and three days for the piles $1(\mathrm{P} 1), 2$ (P2) and 3 (P3), respectively. In the center of P3, 26.87\% moisture was reached in 28 days, while P1 and P2 showed a similar humidity in 37 and 34 days, respectively. After 40 days of processing, the moisture content in the center and the surface of the three piles
\end{abstract}


was stabilized in approximately $20 \%$ and $10 \%$, respectively. P1 and P2 presented a temperature over $40{ }^{\circ} \mathrm{C}$ in the center in 34 days, while $\mathrm{P} 3$ did not exceed $40{ }^{\circ} \mathrm{C}$ after the 26th day. Then, as the dehydration in P3 became faster, the microbial activity in the thermophilic phase lasted eight days less than in the other two treatments. The combustion heat of the material in $\mathrm{P} 3$ was $15852.67 \mathrm{~kJ} / \mathrm{kg}$, therefore it has the quality of waste to be combusted according to the European Union for Responsible Incineration and Treatment of Special Waste. Consequently, pile 3, which had the highest turnover frequency, presented the shortest time for the biodrying of organic waste.

\section{INTRODUCCIÓN}

El biosecado se puede definir como un proceso donde se remueve el agua de los residuos sólidos, aprovechando la actividad metabólica de los microorganismos presentes de manera natural en los residuos. Las reacciones exotérmicas que se presentan durante la biodegradación de la materia orgánica, conllevan un aumento de la temperatura que favorece la eliminación del agua por evaporación. Lo anterior incrementa el poder calorífico de la biomasa permitiendo que se aproveche como combustible alterno (Velis et al. 2009, Zhao et al. 2011). En varios países de Europa, este proceso ya se aplica con el objetivo de reducir la humedad de los residuos sólidos urbanos (RSU) antes de someterlos a incineración. El biosecado en plantas de tratamiento de RSU puede durar entre 13 y 18 días (Zhao et al. 2011), y al final del proceso se obtiene un material con contenido de humedad final de alrededor de $20 \%$, condición necesaria para la recuperación de energía por incineración (Velis et al. 2009).

La mayoría de los trabajos de investigación en biosecado se han enfocado a estudiar este proceso en los RSU dentro de reactores con aireación forzada en sistemas que pueden ser cerrados o abiertos, de pasillos-túneles o tambores rotatorios (Velis et al. 2009, Bilgin y Tulun 2015, Colomer-Mendoza et al. 2016). Algunos trabajos más se han enfocado al biosecado de lodos de plantas de tratamiento de aguas residuales (Navaee-Ardeh et al. 2010, Cai et al. 2013, Villegas y Huiliñir 2014) y de biosecado de residuos de alimentos también en reactores (Mohammed et al. 2017, Yuan et al. 2018).

A nivel industrial, el biosecado también podría aplicarse al tratamiento y aprovechamiento de residuos agrícolas, agroindustriales y forestales, con el objetivo de obtener un combustible alterno. Sin embargo, la literatura sobre esta línea de investigación es muy escasa y el proceso de biosecado en pilas no se ha caracterizado completamente. Robles-Martínez et al. $(2012,2013)$ han realizado pruebas de biosecado de residuos verdes mezclados con cáscara de naranja, obteniendo una disminución del contenido de humedad de 97 y $90 \%$, mientras que ColomerMendoza et al. (2012) obtuvieron una disminución de humedad hasta del $87 \%$. La particularidad de estos experimentos es que se han realizado en pilas al interior de estructuras tipo invernadero, con el objetivo de aprovechar la energía solar y hacer más eficiente el biosecado. En los casos de biosecado en que las pilas no cuentan con ductos de aireación forzada, el suministro de aire y oxígeno al interior de éstas se lleva a cabo mediante volteos manuales, que también sirven para homogeneizar la mezcla de residuos.

Robles-Martínez et al. (2012) y ColomerMendoza et al. (2012) identificaron dos fases en el biosecado operado al interior de una estructura tipo invernadero: $a$ ) una fase de disminución constante de humedad de los residuos (desde $95 \%$ hasta $18 \%$ ) debido a la actividad microbiana y el calor metabólico liberado, la cual ocurre al comienzo del proceso (primeros 16 días) y $b$ ) una fase de secado lento atribuido a fenómenos físicos (convección natural y radiación solar), la cual requiere de mayor tiempo (19 días finales) para remover sólo un $9 \%$ de humedad (desde $18 \%$ hasta $9 \%$ ). Actualmente, en América Latina no se aplica el biosecado para el tratamiento de residuos sólidos orgánicos. El único tratamiento aerobio empleado es el composteo, que se realiza en pilas al aire libre. Por esta razón la fase experimental del presente trabajo se llevó a cabo en pilas (y no en reactores), dado que para los municipios o pequeñas empresas es la forma más sencilla y económica de llevar a cabo estos bioprocesos. El objetivo del presente trabajo es aportar información sobre el efecto de la frecuencia del volteo en el biosecado realizado en pilas semiestáticas de residuos sólidos orgánicos al interior de una estructura tipo invernadero.

\section{MATERIALES Y MÉTODOS}

Se evaluó el efecto de la frecuencia de volteo en el biosecado de tres pilas de $350 \mathrm{~kg}$ cada una, las 
cuales fueron conformadas por una mezcla de residuos orgánicos compuesta por cáscaras de naranja, residuos de poda triturados (mantillo, que funcionó como agente estructurante) y pasto. La frecuencia de volteo fue cada 3, 6 y 10 días para las pilas 3,2 y 1 , respectivamente.

El peso, composición y frecuencia de volteo se presentan en el cuadro I. El proceso de biosecado de las pilas se llevó a cabo al interior de una estructura tipo invernadero, pero sin control de ninguna variable ambiental, es decir, una estructura tubular en forma de casa $(8 \times 16 \times 4$ m de altura $)$, cubierta completamente con plástico de baja densidad con el objetivo de proteger las pilas de la lluvia. El invernadero estuvo asentado en un espacio abierto y en las condiciones ambientales y climáticas de la Ciudad de México durante los meses de septiembre a diciembre. Las condiciones que se monitorearon dentro y fuera de la estructura tipo invernadero fueron temperatura y humedad relativa del aire. Estas mediciones se monitorearon día y noche, cada $2 \mathrm{~h}$, durante el tiempo que duró el bioproceso.

CUADRO I. COMPOSICIÓN DE LAS PILAS DE RESIDUOS

\begin{tabular}{|c|c|c|c|c|c|c|}
\hline & \multicolumn{2}{|c|}{ Pila 1} & \multicolumn{2}{|c|}{ Pila 2} & \multicolumn{2}{|c|}{ Pila 3} \\
\hline & $\begin{array}{l}\text { peso } \\
(\mathrm{kg})\end{array}$ & $\%$ & $\begin{array}{l}\text { peso } \\
(\mathrm{kg})\end{array}$ & $\%$ & $\begin{array}{l}\text { peso } \\
(\mathrm{kg})\end{array}$ & $\%$ \\
\hline \multicolumn{6}{|l|}{ Cáscara de } & 56 \\
\hline Mulch & 79 & 23 & 89 & 25 & 89 & 25 \\
\hline Pasto & 58 & 17 & 68 & 19 & 68 & 19 \\
\hline Total & 337 & 100 & 357 & 100 & 357 & 100 \\
\hline $\begin{array}{l}\text { Frecuencia de } \\
\text { volteo }\end{array}$ & \multicolumn{2}{|c|}{10 días } & \multicolumn{2}{|c|}{6 días } & \multicolumn{2}{|c|}{3 días } \\
\hline
\end{tabular}

Las pilas se conformaron con la forma geométrica de una pirámide. Se estableció que las dimensiones de la altura $(0.65 \mathrm{~m})$ y el ancho $(1.4 \mathrm{~m})$ de la pila se mantuvieran fijas durante todo el proceso. El largo inicial de la base fue de $2 \mathrm{~m}$, mientras que las dimensiones iniciales de la cara superior fueron $1.1 \mathrm{~m}$ de largo y $0.6 \mathrm{~m}$ de ancho. Debido a la deshidratación y degradación parcial de la materia orgánica (MO), el largo de la pila varió conforme avanzó el proceso para mantener la altura y ancho inicial de la pila. Estas dimensiones se midieron cada siete días y el volumen fue calculado con la ecuación 1(Baldor 2004). El esquema de las pilas se presenta en la figura 1.

$$
\begin{aligned}
& V=h / 3[A+a+\sqrt{(A * a)}] \\
& a=A B \times L B ; \quad A=A S \times L S
\end{aligned}
$$

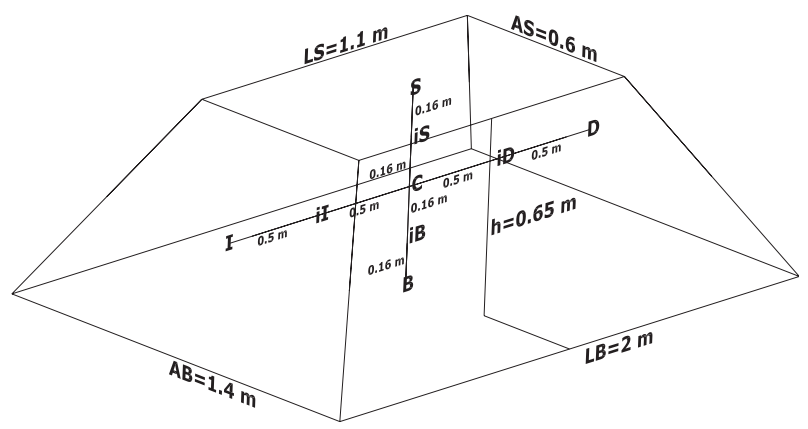

Fig. 1. Esquema de una pila de residuos y distribución de termopares. (C) centro, (D) derecha, (I) izquierda, (S) superior, (B) base, (i) intermedio

\section{Determinación de humedad}

La determinación se hizo cada dos días. El análisis fue realizado por triplicado a partir de muestras de $300 \mathrm{~g}$, las cuales se tomaron en el centro y en la superficie de cada una de las pilas. Se hizo la determinación de contenido de humedad por el método gravimétrico según la metodología reportada por Sadzawka et al. (2005).

\section{Determinación de temperatura y humedad rela- tiva del aire}

El registro de la temperatura se llevó a cabo cada $2 \mathrm{~h}$ durante las $24 \mathrm{~h}$ del día a lo largo de todo el proceso. Se emplearon termopares conectados a una computadora equipada con el programa Opto 22. Se registraron las temperaturas de nueve puntos a distintas profundidades, tanto para el eje vertical (puntos $\mathrm{S}$, iS, C, iB y B) como para el eje horizontal (puntos I, iI, C, iD y D). La separación de los termopares en el eje vertical fue de $0.16 \mathrm{~m}$ y de $0.5 \mathrm{~m}$ a lo largo del eje horizontal. En la figura 1 se indica la colocación de los termopares.

La humedad relativa del aire, dentro y fuera del invernadero, fue registrada diariamente cada $2 \mathrm{~h}$ con el equipo Rainwise Portable Weather Station 99755-06, que mide del 0 a100 \% con una precisión de $\pm 1 \%$.

\section{RESULTADOS Y DISCUSIÓN}

\section{Humedad}

La humedad inicial de las pilas fue de $68 \%$, la cual fue disminuyendo tanto en el centro como en la 
superficie de las pilas conforme avanzó el proceso (Fig. 2). La pérdida de agua en las pilas puede darse en forma líquida (lixiviados) o en forma de vapor de agua. En este trabajo no se registró la formación de lixiviados, por lo que se considera que la disminución de la humedad se debió básicamente a la evaporación de ésta. Los valores de humedad de la superficie fueron menores a los del centro en cualquier tiempo en las tres pilas, porque la superficie está todo el tiempo en contacto directo con el aire del ambiente y este contacto facilita el secado. El contenido final
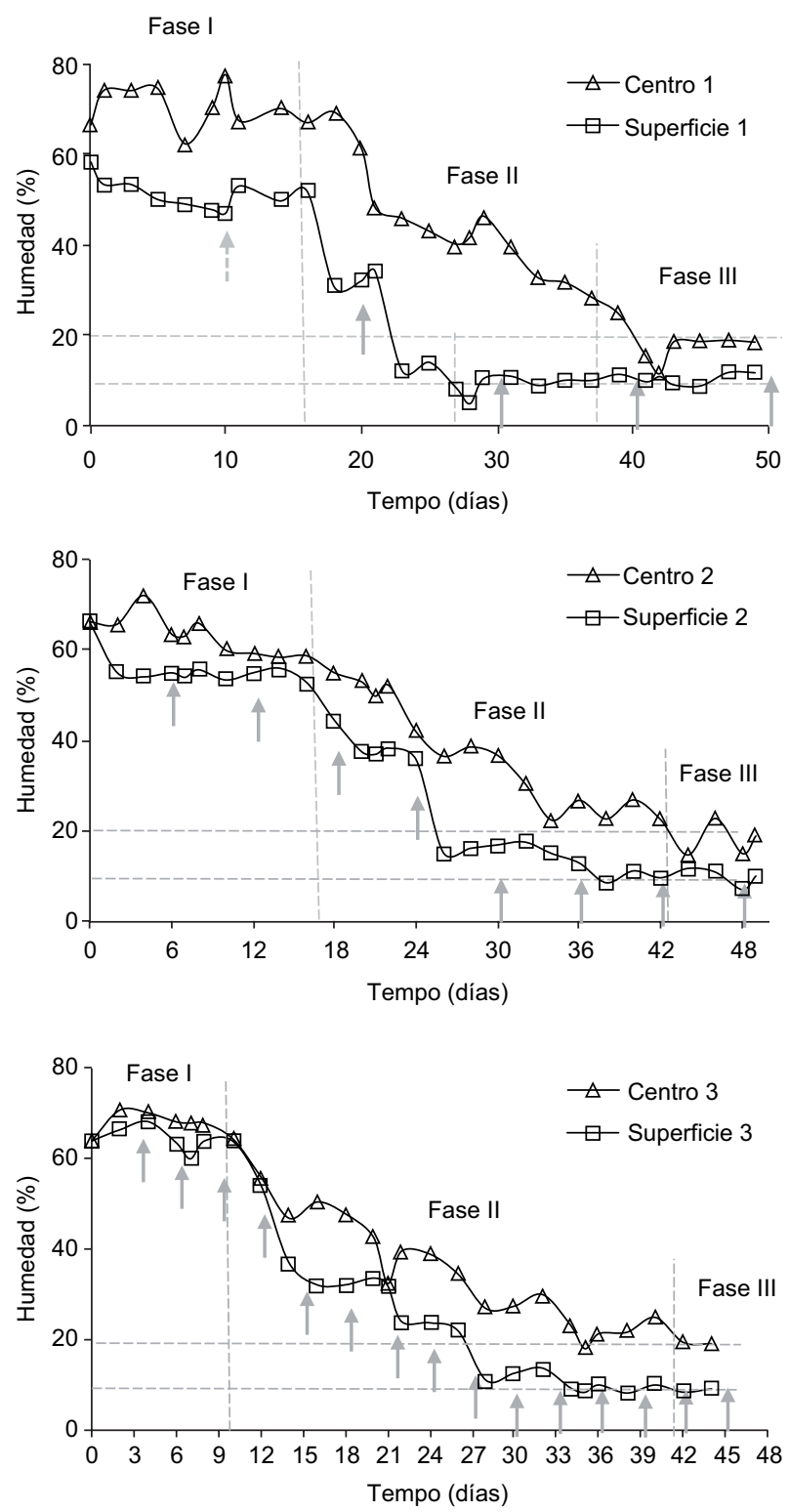

Fig. 2. Comportamiento de la humedad de las pilas de residuos $(1,2$ y 3$)$ durante el biosecado. ( $\uparrow)$ tiempo de volteo de humedad en el centro fue de $20 \%$ y $10 \%$ en la superficie de las tres pilas, lo que indica que dicho centro necesita un proceso más largo para alcanzar la mínima humedad. Lo anterior se puede visualizar después del día 40, cuando la humedad del centro de las pilas sigue bajando muy lentamente.

Colomer-Mendoza et al. (2012) reportan una humedad final de $35 \%$ en un tiempo de proceso de 20 días, para una pila compuesta por residuos verdes y agrícolas con un contenido inicial de humedad de 55 a $80 \%$, mientras que Zhang et al. (2018) reportan una humedad final de $43.2 \%$ (62.3\% de humedad inicial) para el mismo tiempo de proceso de biosecado de aserrín y lodos. Se puede alcanzar una humedad más baja si las pilas son de menor peso, como reportan Robles-Martínez et al. (2012), quienes obtuvieron un contenido final de humedad de $6-10 \%$ en pilas de residuos hortofrutícolas (humedad inicial de $90 \%$ ). Por su parte, Raksanau y Maneechot (2017) informaron que la humedad final fue de $8.41 \%$ en 15 días en pilas de residuos sólidos urbanos sometidos a tratamiento mecánico biológico $(27.39 \%$ de humedad inicial). Como se puede observar, el tiempo de proceso para alcanzar una determinada humedad final, estará determinado en buena medida por el contenido inicial de humedad, origen y naturaleza de los materiales a biosecar.

Se pueden esquematizar tres fases durante la reducción de humedad de las pilas de residuos, como se muestra en la figura 2. Para explicar el comportamiento de estas fases de forma cualitativa, puede aplicarse la ecuación general de balance (Geankoplis 1998):

Acumulación $=$ entrada - salida +
generación - consumo

Con base en este balance, el centro de las pilas se rige por la salida (pérdida de agua durante el biosecado), la generación (vapor de agua producto de la respiración microbiana, que escapa al ambiente convirtiéndose en una salida), y la entrada, que sólo aplica durante el volteo: el vapor de agua, generado por el biosecado como el de la respiración microbiana, sufre una condensación por el enfriamiento del material causado por el volteo del mismo. Como la temperatura de bulbo húmedo del aire puede considerarse igual a la temperatura de la pila, entonces parte del vapor puede condensarse fácilmente si la pila sufre cualquier enfriamiento, es decir, la condensación puede ocurrir si la temperatura de la pila durante el volteo es inferior a la temperatura de bulbo húmedo. Así, el centro sólo está regido por la velocidad de secado, y ésta depende de las condiciones 
ambientales (temperatura, humedad, dirección y velocidad del aire), de la temperatura de la pila (que depende principalmente de la actividad microbiana) y de la frecuencia de volteo.

Mientras que la superficie se rige por la entrada, salida y generación, la entrada se explica porque durante el volteo se mezcla prácticamente la totalidad de la pila (centro y capas intermedias de mayor humedad más la condensación) con la superficie, que tiene menor humedad. Por ello, el centro no se ve afectado después del volteo, mientras que la superficie queda con mayor humedad, lo cual se ve reflejado en pequeños incrementos de humedad durante el proceso de biosecado (Fig. 2).

\section{Fase I}

La primera fase de la pila 3, de 10 días, es la más corta, porque la mayor frecuencia de volteo provoca que la pérdida de humedad sea mayor que en las otras dos pilas, y también que la humedad de la superficie sea muy parecida a la del centro por una mayor frecuencia de mezclado. Por otra parte, una menor frecuencia de volteo aumenta la fase I a 17 días en las pilas 1 y 2 , y ocasiona que la humedad de las superficies sea cada vez más baja que la de los centros en cualquier tiempo que se compare.

La humedad del centro en la pila 1 disminuye poco porque la velocidad de secado es baja, ya que parte del calor generado por la actividad microbiana se usa para calentar la pila (Fig. 3) y el resto para la evaporación de agua. Después de que se efectúa el primer volteo (día 10), la humedad se eleva rápidamente en el centro debido a la condensación parcial del vapor (como ya se explicó líneas arriba), causada por el enfriamiento drástico que sufre la pila durante el volteo (de 65 a $42{ }^{\circ} \mathrm{C}$ ) (Fig. 3). Estos mismos fenómenos ocurren en los centros de las pilas 2 y 3 , pero una frecuencia de volteo mayor produce también una mayor pérdida de humedad, como lo muestran los resultados.

La velocidad de eliminación de humedad de la superficie de las tres pilas es prácticamente de la misma proporción, y una mayor frecuencia de volteo provoca que la humedad de la superficie sea más cercana a la del centro por efecto del mezclado.

\section{Fase II}

En esta fase la temperatura de la pila se equilibra, es decir, ya no sigue aumentando porque se igualan las velocidades de generación de calor microbiano y de enfriamiento de la pila por convección natural, debido a que su temperatura $\left(65-70^{\circ} \mathrm{C}\right)$ es superior a la del ambiente dentro del invernadero, $40-45^{\circ} \mathrm{C}$ en el día y $10-15^{\circ} \mathrm{C}$ en la noche.
A medida que avanza la fase II, la población microbiana se incrementa respecto de la inicial, ya que tiene las condiciones idóneas (materia orgánica y humedad) para seguir creciendo; por lo tanto, habrá mayor consumo de oxígeno. Cada vez que se aplica el volteo (flechas en la Fig. 2) la humedad se eleva (en relación con la humedad del material antes del volteo) por condensación del vapor, debido al enfriamiento de la pila durante el mezclado, de tal forma que una mayor frecuencia de volteo provoca mayor número de altibajos en la humedad de las pilas. Conforme aumenta la frecuencia de volteo se va alargando el tiempo en el cual se alcanza la humedad final del centro de las pilas (40 días para la pila 1 y 44 días para las pilas 2 y 3 ). El centro de la pila 1 presentó una velocidad de biosecado apenas superior que la de los centros de las pilas 2 y 3 , que a su vez presentaron velocidades muy parecidas. Se esperaba que un mayor volteo provocara mayor velocidad de biosecado por mayor aireación, pero al mismo tiempo un mayor volteo provoca la condensación de una parte del vapor por enfriamiento de la pila, de tal manera que el efecto neto es una postergación del tiempo para llegar a la humedad final. Navaee-Ardeh et al. (2006) y Robles-Martínez et al. (2012) no reportan estos altibajos porque no hubo volteo en sus trabajos.

Esta misma explicación aplica para el comportamiento de la humedad en la superficie de las pilas, pero ahora el efecto es más pronunciado. La velocidad de secado de la superficie de las pilas es mayor que la del centro (Fig. 2) porque la superficie está en contacto directo con el aire fresco, facilitando con esto la perdida de humedad. También se puede apreciar que la velocidad de secado de la superficie de la pila 1 es la más alta, seguida de la pila 2 , y la velocidad más baja es la de la pila 3. Asimismo, una mayor frecuencia de volteo alarga el tiempo de secado final de las superficies, de 27 días para la pila 1 , a 36 días para las pilas 2 y 3 . De este modo, si la superficie de la pila 1 no se hubiera mezclado se hubiera secado más rápidamente, y un mayor mezclado, con un centro húmedo y con condensación parcial, equivale a "humedecer" la superficie; como consecuencia, el tiempo para alcanzar el secado final en la superficie de la pila se posterga.

\section{Fase III}

La humedad de la superficie de las pilas alcanza un contenido final de $10 \%$ y luego permanece invariable, lo que muestra que dicha humedad ya está en equilibrio con la del ambiente, por lo tanto la frecuencia de volteo ya no tiene ningún efecto en el secado. Para el material que se encontraba en 

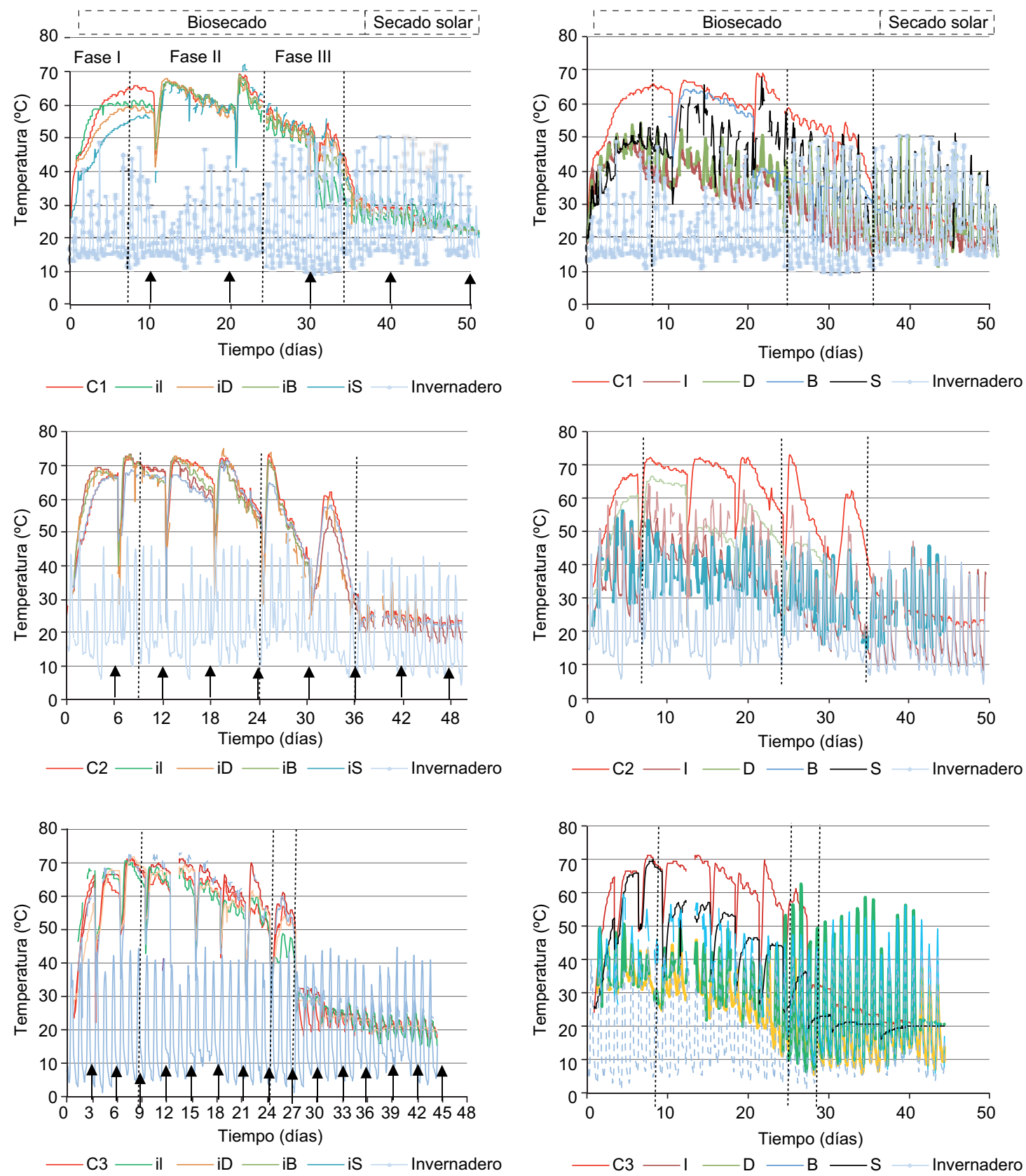

Fig. 3. Perfil de temperaturas del centro $(\mathrm{C} 1, \mathrm{C} 2, \mathrm{C} 3)$, capas internas (iI, iD, iB, iS) y caras laterales $(I, D, B, S)$ de las pilas durante el biosecado. $(\uparrow)$ punto de volteo

el centro de las pilas 2 y 3 hicieron falta más de 50 días para llegar a este mismo valor de humedad. La velocidad de secado del centro de las pilas es mucho más baja en comparación con la fase II, y la frecuencia de volteo afecta poco, porque el contenido de humedad es tan bajo (y por ende el oxígeno puede ser insuficiente) que inactiva el metabolismo microbiano. Finalmente, sólo queda el ambiente (temperatura y humedad ambiental y radiación solar) como regidor del secado.

Este análisis permite concluir que las fases I y II son regidas por el biosecado (ambiente y actividad microbiana alta en la primera fase; ambiente y metabolismo limitado y descendente en la fase II), y la frecuencia de volteo ayuda a la eliminación de humedad a través de la aireación, pero también 
CUADRO II. VELOCIDADES DE SECADO DE LAS PILAS DE RESIDUOS

\begin{tabular}{lcccccccc}
\hline & \multicolumn{3}{c}{ CENTROS } & & \multicolumn{3}{c}{ SUPERFICIES } \\
\cline { 2 - 3 } \cline { 6 - 8 } & Pila 1 & Pila 2 & Pila 3 & & Pila 1 & Pila 2 & Pila 3 \\
\cline { 2 - 3 } \cline { 6 - 8 } & \multicolumn{2}{c}{ kg agua eliminada/día } & & \multicolumn{2}{c}{ kg agua eliminada/día } \\
\hline Fase I & 0.40 & 1.68 & 1.47 & & 1.59 & 1.47 & 1.79 \\
Fase II & 6.26 & 5.49 & 4.52 & & 13.06 & 7.89 & 4.57 \\
Fase III & 0.00 & 1.19 & 0.79 & & 0 & 0 & 0 \\
Total 49 días & 4.58 & 4.55 & 4.39 & & 4.63 & 4.64 & 4.49 \\
\hline
\end{tabular}

"aporta" agua al sistema por condensación parcial, retardando el biosecado. Por su parte, la fase III sólo es regida por el secado solar (ambiente) y el volteo facilita el secado, pero en esta fase el efecto del volteo no es significativo porque la fuerza impulsora para el secado es muy baja debido al bajo contenido de humedad de las pilas (menor al $20 \%$ ).

Finalmente, en el cuadro II, se muestran las velocidades de secado calculadas con los resultados mostrados en esta sección del trabajo. Claramente se observa que en la fase II se lleva a cabo prácticamente todo el secado de las pilas, tanto en el centro como en la superficie. La mayor velocidad de secado en esta fase se presenta en la pila 1 (centro y superficie), que es la de menor frecuencia de volteo.

\section{Temperatura}

En la figura 3 se muestran los resultados del comportamiento de la temperatura en las pilas de residuos. El proceso global puede dividirse en dos etapas: biosecado y secado ambiente. La etapa de biosecado tuvo una duración de 36 días y se puede dividir en tres fases, mientras que la etapa de secado ambiente tuvo una duración de 14 días.

Se puede observar que el centro y las capas internas de cada pila $(\mathrm{C}, \mathrm{iI}, \mathrm{iD}, \mathrm{iB}, \mathrm{iS})$ registraron las mayores temperaturas, seguidos por la cara base (B), que no tiene salida de calor, después por la cara superior (S), que recibe directamente la radiación solar, y al final por las caras laterales (I y D), que están en contacto con el ambiente del invernadero. Lo anterior concuerda con lo reportado por Rada et al. (2007), quienes también afirmaron que el centro de la pila era el punto más caliente y las superficies las de menor temperatura.

Para explicar cualitativamente los perfiles de temperatura de las pilas es necesario aplicar la ecuación general de balance (ecuación 2), que lleva al siguiente análisis: la etapa de biosecado se rige por la generación (actividad microbiana), entrada (radiación solar) y salida de calor (enfriamiento por convección natural), mientras que la etapa de secado ambiente se rige por la entrada (radiación solar) y salida (enfriamiento por convección natural).

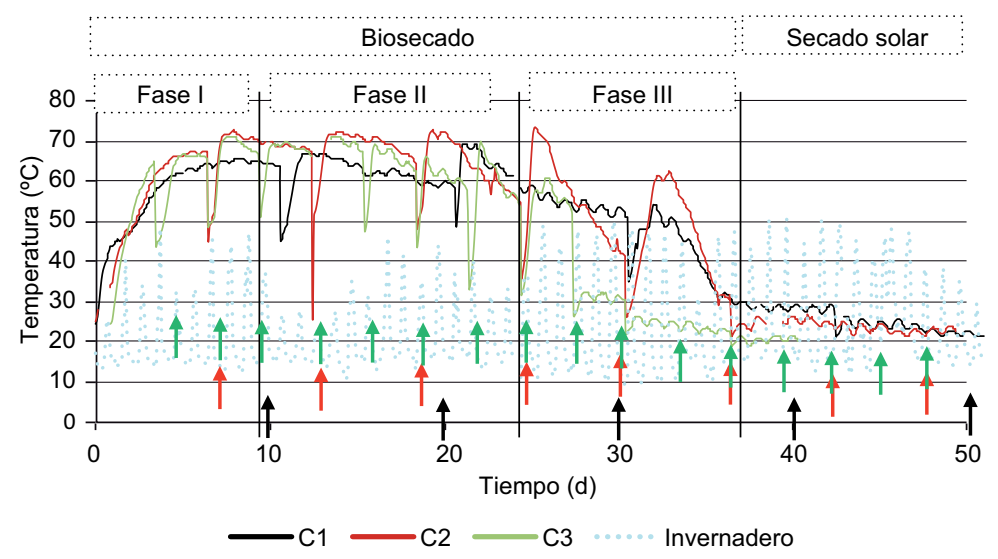

Fig. 4. Perfil de temperaturas del centro de las pilas(1, 2 y 3$)$ durante el biosecado. $(\uparrow)$ punto de volteo 
La frecuencia de volteo influye positivamente estos términos de la ecuación.

A continuación se explicará el comportamiento de los perfiles de temperatura tomando como base la pila 1, pero el análisis y conclusiones que se derivan de dicho análisis se aplican a los perfiles de las pilas 2 y 3 .

\section{Biosecado fase I}

La primera fase dura ocho días (Fig. 3) y consiste en el rápido crecimiento de la población microbiana, lo cual provoca que la generación de calor supere su salida; por lo tanto, la temperatura se va elevando a partir de la inicial $\left(26^{\circ} \mathrm{C}\right)$. Después de esta elevación se alcanzó un máximo $\left(65^{\circ} \mathrm{C}\right.$ : centro y capas internas; $53{ }^{\circ} \mathrm{C}$ : caras laterales) porque la salida de calor por convección natural (que siempre está actuando) equilibra la generación.

La temperatura de las caras laterales (I y D) presentan altibajos más pronunciados que los del centro, es decir, $45-50{ }^{\circ} \mathrm{C}$ durante el día y $25-35^{\circ} \mathrm{C}$ durante la noche, debido a que las superficies están en contacto directo con el aire fresco y éste tiene un efecto marcado en las temperaturas registradas. Cabe destacar que la base de la pila (B) se comporta como si fuera una capa interna debido a que el calor sólo tiene salida hacia arriba y hacia los lados, por lo que sus temperaturas son altas en esta fase. La cara superior (S) también se comporta como capa interna con altas temperaturas, porque recibe directamente la radiación solar. Por su parte, las caras laterales $\mathrm{B}$ y $\mathrm{S}$ de las pilas 2 y 3 no presentan temperaturas tan altas como las de la pila 1 por el mayor número de volteos, y nuevamente las caras I y D son las más "frías", como en la pila 1.

Cuando se comparan las temperaturas de los centros de la pilas en esta primera fase se obtienen los resultados mostrados en la figura 4. Claramente se observa que las temperaturas de las pilas 2 y 3 , que son parecidas entre sí, son más altas en tres a cuatro grados que las temperaturas de la pila 1. Una mayor frecuencia de volteo produce temperaturas más elevadas porque causa mayor oxigenación, y ésta provoca mayor generación de calor de la población microbiana que consume el oxígeno para degradar la materia orgánica. Se observó que frecuencias de tres y seis días en el volteo provocan los mismos niveles de temperatura.

\section{Biosecado fase II}

Esta fase está regida por el equilibrio entre la generación y la salida de calor (Fig. 3), provocando que la temperatura se mantenga en valores altos por un determinado periodo (días ocho a 24).
Cuando se aplica el primer volteo (día 10) el centro y las capas internas se enfrían rápidamente de 65 a $42{ }^{\circ} \mathrm{C}$ (las superficies se enfrían de 53 a $32{ }^{\circ} \mathrm{C}$ ) debido a la transferencia de calor hacia el ambiente; después la temperatura vuelve a elevarse rápidamente (en un día) hasta el valor máximo debido a la intensa actividad microbiana de una población mayor que fue oxigenada con el volteo (un comportamiento similar es reportado por Zambra et al. 2011).

Este volteo también ocasiona pérdida de humedad, y el oxígeno apenas suministrado se consume rápidamente, quedando en un nivel tal que empieza a limitar la actividad microbiana; esta limitación provoca que la generación de calor ya no sea tan intensa como al principio $\mathrm{y}$, al ser contrarrestada por la salida de calor, se observa que la temperatura del centro y capas internas desciende lentamente de 67 a $57^{\circ} \mathrm{C}$ durante los siguientes 10 días. En las superficies, la temperatura desciende de 50 a $35^{\circ} \mathrm{C}$; a su vez, éstas registran $50{ }^{\circ} \mathrm{C}$ durante el día y $35^{\circ} \mathrm{C}$ durante la noche.

En el segundo volteo (día 20) sucede exactamente lo mismo que acaba de explicarse, pero ahora la limitación por falta de oxígeno es más significativa (Zhang et al. 2009) porque el contenido de humedad sigue disminuyendo y se esperaría que la población microbiana siguiera aumentando. La generación de calor es ahora menos intensa y por ende la temperatura disminuye más rápidamente que en el primer volteo. El centro y las capas internas bajan de 67 a $50^{\circ} \mathrm{C}$ durante los siguientes diez días (las superficies disminuyen de 50 a $22^{\circ} \mathrm{C}$ en el mismo periodo).

Ahora, si se comparan las temperaturas de los centros de las pilas en esta fase II se pueden observar los resultados de la figura 4. Nuevamente, como en la fase I, se observa que las temperaturas de las pilas 2 y 3 , que son similares entre sí, son más altas en cinco a nueve grados que las temperaturas de la pila 1. Entonces, una mayor frecuencia de volteo produce temperaturas más elevadas (Oviedo et al. 2014). También se puede afirmar que las frecuencias de $3 \mathrm{y}$ 6 días en el volteo producen los mismos niveles de temperatura.

\section{Biosecado fase III}

Esta fase (días 24 a 36) está gobernada por bajos contenidos de humedad (la cual es el vehículo del oxígeno atmosférico y este último a su vez es consumido por una población microbiana cada vez mayor), lo que ocasiona una limitación de la actividad microbiana. Dicha limitación se va incrementando a medida que la humedad sigue disminuyendo como producto del biosecado de las pilas. 
En el tercer volteo (día 30) sucede lo ya comentado: la temperatura del centro y capas internas baja rápidamente de 50 a $32{ }^{\circ} \mathrm{C}$ por enfriamiento (las superficies descienden de 45 a $20^{\circ} \mathrm{C}$ ), se oxigena la población microbiana aumentando la generación de calor, que causa nuevamente un rápido ascenso de la temperatura a $52{ }^{\circ} \mathrm{C}$ (las superficies suben a $45^{\circ} \mathrm{C}$ ). Ahora el contenido de humedad (que disminuye de 40 a $25 \%$ ) limita la actividad microbiana de una población cada vez mayor, provocando que la velocidad de generación de calor sea la más baja de todo el proceso y, en consecuencia, que la disminución de temperatura sea aún más rápida que en las dos fases anteriores: de 52 a $30{ }^{\circ} \mathrm{C}$ en un periodo de seis días. La primera disminución de temperatura es de 67 a $57{ }^{\circ} \mathrm{C}$ en 10 días, con una baja de humedad de 68 a $60 \%$; la segunda reducción es de 67 a $50{ }^{\circ} \mathrm{C}$, también en 10 días, con una baja de humedad de 60 a $40 \%$, mientras que en esta última fase se registra un descenso de $18^{\circ} \mathrm{C}$ en seis días.

Cuando se comparan las temperaturas de los centros de pila (Fig. 4), la pila 2 eleva su temperatura hasta $10^{\circ} \mathrm{C}$ más que la pila 1 después de cada volteo, como puede observarse en los resultados, debido a la mayor oxigenación que recibe, producto de una mayor frecuencia de volteo.

En cambio se esperaría que la pila 3, que es la de mayor volteo, tuviera temperaturas similares a las de la pila 2; sin embargo, esto no ocurre porque la frecuencia de volteo a la cual fue sometida propició el menor contenido de humedad en el menor tiempo, por lo que aun con mayor suministro de oxígeno carece de la humedad necesaria. Esto ocasiona que la actividad microbiana cese por completo; en consecuencia, la generación de calor es nula. Inclusive después de cada volteo en los días 27, 30, 33 y 36, la temperatura no se eleva de acuerdo con los esperado y permanece relativamente baja (con un valor promedio de $28^{\circ} \mathrm{C}$ ). Así, la mayor frecuencia de volteo reduce en $75 \%$ la duración de la fase III en el centro de la pila 3 .

\section{Secado ambiente}

Esta etapa (Fig. 3) está controlada por la temperatura del invernadero porque ya no existe generación significativa de calor microbiano (días 37 a 40). Por la noche la temperatura del invernadero es de $15^{\circ} \mathrm{C}$ y el centro y capas internas tienden a enfriarse, disminuyendo sólo de 30 a $28{ }^{\circ} \mathrm{C}$ (las superficies registran $17^{\circ} \mathrm{C}$ ).

Durante el día la temperatura del invernadero es de $50{ }^{\circ} \mathrm{C}$ (resultado de la radiación solar), por lo que el centro y capas internas tienden a calentarse, pero sólo aumentan de 28 a $30^{\circ} \mathrm{C}$, debido a que la penetración de calor hacia el centro y las capas intermedias es lenta por la distancia que tiene que penetrar durante el periodo de sol de 8:00 a 19:00 horas (las superficies se calientan a $40{ }^{\circ} \mathrm{C}$ ).

Cuando se aplica el volteo (día 40) el centro y las capas intermedias se enfrían a $25^{\circ} \mathrm{C}$; después ya no sufren ningún aumento de temperatura porque no hay actividad metabólica por falta de humedad. Por la noche desciende a $23^{\circ} \mathrm{C}$ y durante el día se eleva otra vez a $25^{\circ} \mathrm{C}$.

Por otro lado, al estar en contacto directo con el aire del invernadero, las superficies (I, D, S) registran $40{ }^{\circ} \mathrm{C}$ durante el día y $18{ }^{\circ} \mathrm{C}$ durante la noche (la temperatura del invernadero varía de 50 a $15{ }^{\circ} \mathrm{C}$ del día a la noche, respectivamente).

En el caso de la temperatura de los centros (Fig. 4), las pilas 1 y 2 presentan temperaturas semejantes entre sí, las cuales bajan de 30 a $23{ }^{\circ} \mathrm{C}$ en 14 días. Este mismo comportamiento ya fue explicado anteriormente para la pila 1 .

En el caso de la pila 2, después de que se aplica el sexto volteo (día 36) la temperatura baja de 30 a $27^{\circ} \mathrm{C}$. Luego desciende a $25^{\circ} \mathrm{C}$ durante la noche y asciende nuevamente a $27^{\circ} \mathrm{C}$ en el día. Estas variaciones permanecen hasta el siguiente volteo porque ya no hay actividad microbiana (en el día 42 se aplica el siguiente volteo y se repite el mismo comportamiento, pero ahora la temperatura varía de 25 a $23^{\circ} \mathrm{C}$ ). En el caso del centro de la pila 3, la de mayor frecuencia de volteo, el comportamiento de la temperatura se puede explicar de la misma forma, pero como se señaló en su momento, en el día 30 alcanza el mínimo de humedad (25\%). Este umbral implica el cese de actividad de la población microbiana; por lo tanto, la generación de calor es nula e inicia el secado solar, que abarca desde este día hasta el día 50 . En esta etapa se tiene un descenso de temperatura de 30 a $22{ }^{\circ} \mathrm{C}$.

\section{CONCLUSIONES}

Los datos evidenciaron que la pérdida de humedad de las pilas en el invernadero se llevó a cabo en dos etapas: biosecado (actividad microbiana y radiación solar) y secado ambiente (sólo radiación solar).

El biosecado ocupa el $72 \%$ del tiempo total del proceso (50 días) si la frecuencia de volteo es de cada 10 días. La investigación también mostró que un volteo realizado cada tres días reduce la etapa de biosecado a sólo $54 \%$ del tiempo total. Los resultados de la investigación mostraron que en el biosecado ocurren todos los cambios significativos 
del proceso. Las pilas alcanzan su peso mínimo, pero un nivel de volteo de cada tres días induce una menor reducción de peso debido a la disminución del tiempo de la actividad microbiana, que es la que degrada la materia orgánica. El $\mathrm{pH}$ alcanza su valor máximo y fue independiente de la frecuencia de volteo. La humedad de las pilas se reduce en $80 \%$, pero un volteo realizado cada tres días aumenta el tiempo para alcanzar la humedad mínima en la superficie, ya que esta última se mezcla con mayor masa y humedad en el centro de la pila. Por el contrario, con tiempos de volteo de 10 días disminuye el periodo en que se alcanza la humedad mínima, lo que sería interesante a nivel semiindustrial, ya que al requerirse menor volteo y menor tiempo de proceso, se necesitaría menos trabajo y, por tanto, se tendría una mayor eficiencia del proceso.

La investigación también evidenció que en la etapa de biosecado se alcanzan las temperaturas más altas del proceso: $65^{\circ} \mathrm{C}$ para el centro de la pila 1. Una frecuencia de volteo de cada tres o seis días provoca temperaturas aun mayores: $71{ }^{\circ} \mathrm{C}$ en el centro de las pilas 2 y 3 , debido a una mayor oxigenación. Las temperaturas de las caras superficiales de las pilas varían de 30 a $55^{\circ} \mathrm{C}$ aproximadamente. Estas temperaturas son dependientes tanto del calor microbiano como de la temperatura dentro del invernadero. La etapa del biosecado termina cuando se acaba la actividad microbiana; a partir de ese momento, la temperatura desciende drásticamente porque ya no hay aporte de calor microbiano.

En la etapa de secado al ambiente, donde ya no hay actividad microbiana, las pilas alcanzan su humedad mínima (14 a 19\%). Las temperaturas del centro, base y capas intermedias son las más bajas del proceso: $27^{\circ} \mathrm{C}$ para la pila de menor frecuencia de volteo, $24{ }^{\circ} \mathrm{C}$ para la pila 2 , y $21{ }^{\circ} \mathrm{C}$ para la pila de mayor nivel de volteo. Estos resultados demuestran, por otra parte, que la radiación solar no tiene la capacidad de calentar estos puntos a pesar de que las temperaturas dentro del invernadero varían de 42 a $49^{\circ} \mathrm{C}$ durante el día (en la noche, estos puntos conservan su temperatura $\left[21 \mathrm{a} 27^{\circ} \mathrm{C}\right]$, a pesar de que la temperatura del invernadero descendió a $7-15^{\circ} \mathrm{C}$ ). Las caras laterales superficiales (I, D, S) registran temperaturas iguales entre sí y prácticamente iguales a la temperatura del invernadero: $49^{\circ} \mathrm{C}$ en el día y $15^{\circ} \mathrm{C}$ en la noche para la pila 1 (septiembre y octubre), y 42 y $7{ }^{\circ} \mathrm{C}$ para las pilas 2 y 3 (meses de noviembre y diciembre), resultados que demuestran que al no haber aporte de calor microbiano, las temperaturas superficiales ya sólo dependen de las condiciones climatológicas del invernadero.

\section{REFERENCIAS}

Baldor J.A. (2004). Geometría plana y del espacio y trigonometría. 20a reimp. Publicaciones Cultural, México, p. 281.

Bilgin M. y Tulun Ş. (2015). Biodrying for municipal solid waste: Volume and weight reduction. Environ. Technol. 36 (13), 1691-1697. DOI: $10.1080 / 09593330.2015 .1006262$

Cai L., Chen T.B., Gao D., Zheng G.D., Liu H.T. y Pan T.H. (2013). Influence of forced air volume on water evaporation during sewage sludge bio-drying. Water Res. 47 (13), 4767-4773. DOI: 10.1016/j.watres.2013.03.048

Colomer-Mendoza F.J., Robles-Martínez F., Herrera-Prats L., Gallardo-Izquierdo A. y Bovea M.D. (2012). Biodrying as a biological process to diminish moisture in gardening and harvest wastes. Environ. Dev. Sustain. 14 (6), 1013-1026. DOI: 10.1007/s10668-012-9369-1

Colomer-Mendoza F.J., Robles-Martínez F., Piña-Guzmán A.B., Pablo-Vicente M. y Gallardo-Izquierdo A. (2016). Influence of different airflows and the presence of bulking agent on biodrying of gardening wastes in reactors. Rev. Int. Contam. Ambie. 32 (Especial Residuos Sólidos), 161-171.

DOI: 10.20937/RICA.2016.32.05.12

Geankoplis C.J. (1998). Procesos de transporte y operaciones unitarias. 3a ed. CECSA, Distrito Federal, México, 1008 pp.

Mohammed M., Ozbay I., Karademir A. e Isleyen M. (2017). Pre-treatment and utilization of food waste as energy source by bio-drying process. International Scientific Conference "Environmental and Climate Technologies", CONECT 2017, Riga (Latvia), 10-12 May. Enrgy. Proced. 128, 100-107.

DOI: 10.1016/j.egypro.2017.09.021

Navaee-Ardeh S., Bertrand F. y Stuart P.R. (2010). Key variables analysis of a novel continuous biodrying process for drying mixed sludge. Bioresource Technol. 101 (10), 3379-3387. DOI: 10.1016/j.biortech.2009.12.037

Oviedo-Ocaña E.R., Marmolejo-Rebellón L.F. y TorresLozada P. (2014). Influencia de la frecuencia de volteo para el control de la humedad de los sustratos en el compostaje de biorresiduos de origen municipal. Rev. Int. Contam. Ambie. 30 (1), 91-100.

Raksanau C. y Maneechot P. (2017). The potential of bio-drying process in improving quality of the processed waste from the mechanical biological treatment for industrial heating systems. Int. J. Renew. Energ. 12 (2), 53-63.

Rada E.C., Franzinelli A., Taiss M., Ragazzi M., Panaitescu V. y Apostol T. (2007). Lower heating value dynamics during municipal solid waste bio-drying. Environ. Technol. 28 (4), 463-469.

DOI: $10.1080 / 09593332808618807$ 
Robles-Martínez F., Silva-Rodríguez E.M., EspinosaSolares T., Piña-Guzmán B., Calixto-Mosqueda C., Colomer-Mendoza F. J. y Durán-Páramo E. (2012). Biodrying under greenhouse conditions as pretreatment for horticultural waste. J. Environ. Prot. 3, 298-303. DOI: $10.4236 /$ jep.2012.34038

Robles-Martínez F., Gerardo-Nieto Ó., Piña-Guzmán A.B., Montiel-Frausto L., Colomer-Mendoza F.J. y OrozcoÁlvarez C. (2013). Obtención de un combustible alterno a partir del biosecado de residuos hortofrutícolas. Rev. Int. Contam. Ambie. 29 (Sup. 3), 79-88.

Sadzawka R.A., Carrasco R.M.A., Grez Z.R. y Mora G.M.L. (2005). Métodos de análisis de compost. Instituto de Investigaciones Agropecuarias, Serie Actas $\mathrm{N}^{\circ}$ 30, Santiago, Chile, $142 \mathrm{pp}$.

Velis C.A., Longhurst P.J., Drew G.H., Smith R. y Pollard S.J.T. (2009). Biodrying for mechanical-biological treatment of wastes: A review of process science and engineering. Bioresource Technol. 100 (11), 2747-2761.

DOI: 10.1016/j.biortech.2008.12.026

Villegas M. y Huiliñir C. (2014). Biodrying of sewage sludge: Kinetics of volatile solids degradation under different initial moisture contents and air-flow rates. Bioresource Technol. 174, 33-41.

DOI: 10.1016/j.biortech.2014.09.136
Yuan J., Li Y., Zhang H., Zhang D., Chadwick D., Li G., Wang G., Chi M. y Yang F. (2018). Effects of adding bulking agents on the biodrying of kitchen waste and the odor emissions produced. J. Environ. Sci. 67, 344355. DOI: $10.1016 /$ j.jes.2017.08.014

Zambra C.E., Moraga N.O. y Escudey M. (2011). Heat and mass transfer in unsaturated porous media: Moisture effects in compost piles self heating. Int. J. Heat Mass Tran. 54 (13-14), 2801-2810.

DOI: 10.1016/j.ijheatmasstransfer.2011.01.031

Zhang D., He P.J. y Shao L. (2009). Effect of leachate inoculum on biopretreatment of municipal solid waste by a combined hydrolytic-aerobic process. J. Environ. Sci. 21 (8), 1162-1168.

DOI: $10.1016 / \mathrm{S} 1001-0742(08) 62397-9$

Zhang H.Y., Krafft T., Gao D., Zheng G.D. y Cai L. (2018). Lignocellulose biodegradation in the biodrying process of sewage sludge and sawdust. Dry Technol, 36 (3), 316-324. DOI: 10.1080/07373937.2017.1326502

Zhao L., Gu W.M., He P.J. y Shao L.M. (2011). Biodegradation potential of bulking agents used in sludge bio-drying and their contribution to bio-generated heat. Water Res. 45 (6), 2322-2330.

DOI: 10.1016/j.watres.2011.01.014 